Utah State University

DigitalCommons@USU

\title{
A Randomized Controlled Trial of Online Acceptance and Commitment Therapy to Improve Diet and Physical Activity Among Adults Who Are Overweight/Obese
}

\author{
Michael E. Levin \\ Utah State University, mike.levin@usu.edu \\ Julie M. Petersen \\ Utah State University, julie.petersen@aggiemail.usu.edu \\ Carrie Durward \\ Utah State University, carrie.durward@usu.edu \\ Brittany Bingeman \\ Utah State University, brittany.bingeman@usu.edu \\ Elizabeth Davis \\ Utah State University, elizabeth.davis@usu.edu \\ Cindy Nelson

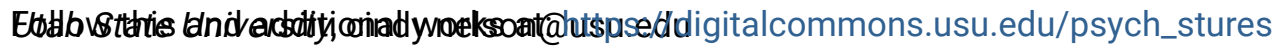 \\ Part of the Psychology Commons
}

See next page for additional autithors

\section{Recommended Citation}

Levin, M.E., Petersen, J.M., Durward, C., Bingeman, B., Davis, E., Nelson, C. \& Cromwell, S. (In Press). A randomized controlled trial of online acceptance and commitment therapy to improve diet and physical activity among adults who are overweight/obese. Translational Behavioral Medicine.

This Article is brought to you for free and open access by the Psychology Student Works at DigitalCommons@USU. It has been accepted for inclusion in Psychology Student Research by an authorized administrator of DigitalCommons@USU. For more information, please contact digitalcommons@usu.edu.

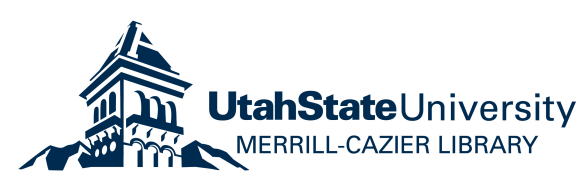


Authors

Michael E. Levin, Julie M. Petersen, Carrie Durward, Brittany Bingeman, Elizabeth Davis, Cindy Nelson, and Shannon Cromwell 
A randomized controlled trial of online acceptance and commitment therapy to improve diet and physical activity among adults who are overweight/obese

Michael E. Levin, Ph.D.*, Julie M. Petersen, B.A., Carrie Durward, Ph.D., Brittany Bingeman, Ph.D., Elizabeth Davis, Ph.D., Cindy Nelson, Ph.D., \& Shannon Cromwell, Ph.D. Utah State University

* Corresponding author. Utah State University, 2810 Old Main Hill, Logan, UT 84322, United States. Phone: +001 (541) 531-3892; Fax: +001 (435) 797-1448, E-mail address:

Mike.Levin@usu.edu.

Funding Sources: "This study was funded by the Utah State University Extension Grants Program."

Conflicts of Interest: "The authors declare that they have no conflicts of interest."

Human Rights: “All procedures performed in studies involving human participants were in accordance with the ethical standards of the institutional and/or national research committee and with the 1964 Helsinki declaration and its later amendments or comparable ethical standards."

Informed Consent: "Informed consent was obtained from all individual participants included in the study."

Acknowledgements: We would like to thank Drs. Suzanne Prevedel and Emma Parkhurst for their help in conducting this study. 


\begin{abstract}
Background: Acceptance and commitment therapy (ACT) has shown benefit for improving diet, physical activity, and weight among adults who are overweight and obese. However, research todate in this area has primarily evaluated ACT delivered through in-person interventions, which has more limited access relative to online formats. Purpose: The present study evaluated an online guided self-help program that integrated ACT with nutrition education to improve healthy eating and physical activity. Methods: A sample of 79 adults who were overweight/obese were randomized to receive the 8-week ACT on Health program plus weekly phone coaching or to a waitlist. Results: Participants completed 5.5 ACT sessions on average (out of 8) and reported moderately high program satisfaction. Participants in the ACT condition improved significantly more than the waitlist at posttreatment on the primary outcome of healthy eating index (HEI; based on 24-hour recall assessments) and almost all secondary outcomes assessing self-reported eating behaviors, weight, mental health, weight self-stigma, and psychological inflexibility. However, no intervention effects were found for self-reported physical activity. At 8-week follow-up, improvements were maintained for most outcomes in the ACT condition, but not for the HEI. Improvements in psychological inflexibility mediated treatment effects on some outcomes, but not HEI or weight. Conclusions: Overall, delivering ACT through online guided self-help combined with nutrition education appears promising for improving healthy eating, weight, and self-stigma, but results for physical activity and long-term behavior change are unclear, possibly due to limitations in the ACT on Health program.
\end{abstract}

Keywords: acceptance and commitment therapy, mindfulness, self-help, obesity, stigma, eHealth. 
A randomized controlled trial of online acceptance and commitment therapy to improve diet and physical activity among adults who are overweight/obese

Obesity is associated with a range of physical health concerns and mortality risks [1]. Diet and physical activity not only contribute to obesity, but also have direct effects on physical health and mortality $[2,3]$. Diet and physical activity, as well as weight loss, continue to be challenging outcomes to improve in behavioral interventions [4-6].

A growing body of research indicates the potential benefits of Acceptance and Commitment Therapy (ACT) [7] for diet, physical activity, and weight loss [8-12]. ACT is a modern cognitive behavior therapy (CBT) that aims to reduce maladaptive behavior regulatory effects of cognition, affect, and other internal experiences (i.e., psychological inflexibility) through acceptance and mindfulness-based methods, while increasing adaptive motivators for behavior through values-based methods [7]. A key difference between ACT and traditional CBT is its emphasis on changing how individuals respond to internal experiences (i.e., reducing the impact of thoughts and feelings on behavior through acceptance and mindfulness), rather than seeking to directly alter internal experience (i.e., change strategies like cognitive restructuring and emotion regulation). When delivered in-person by a therapist, ACT has been found effective for improving diet, physical activity, weight loss, and quality of life among adults who are overweight/obese in several randomized controlled trials [8-12]. Furthermore, studies indicate that ACT improves these outcomes through reductions in psychological inflexibility [10-12].

Yet, the reach of ACT for promoting health among adults who are overweight and obese may be limited due to the in-person format. This format requires the ready availability of providers trained in ACT, who are typically therapists that may not be integrated in the settings 
where individuals seek services. It also requires the ability and willingness for individuals to seek resources in-person, which may be limited by barriers such as cost, travel, time, and stigma.

One recent solution has been to develop self-guided online ACT interventions that people can complete on their own, from the convenience and privacy of their own home. There is a well-established literature regarding the effectiveness of such self-guided online behavioral interventions for weight management and health promotion more broadly [13]. Such research is more preliminary with ACT as a newer intervention applied to this area. Preliminary RCTs have found initial support for self-guided ACT improving physical activity and/or eating behaviors in book [14], mobile app [15], and DVD formats [16].

However, there are some gaps in research on self-guided ACT for weight-related concerns. Prior self-guided ACT interventions have not integrated ACT with nutrition education, which may be ideal in providing behavioral strategies to increase specific dietary changes while using ACT to address psychological barriers and motivation for change. Similarly, most interventions have not targeted weight self-stigma, which has been found to impede adherence to dietary and physical activity changes in health promotion interventions $[17,18]$, and to improve from ACT-based self-help books [14] and workshops [11]. Finally, most ACT interventions have been purely self-guided, but research suggests greater adherence and effectiveness when they include guidance from a phone coach [19], and phone coaching has been found to provide additional benefits when added to an ACT self-help book [14].

The current waitlist-controlled RCT sought to evaluate ACT on Health, which integrated nutrition education, physical activity goal setting, and ACT in an 8-session online guided selfhelp course with brief, weekly phone coaching to improve program adherence. Study predictions included that 1) ACT on Health would be acceptable to participants (adults who are 
overweight/obese) as indicated by a high degree of program engagement and high self-reported program satisfaction; 2) participants assigned to ACT would improve on self-reported healthy eating, physical activity, weight, weight self-stigma, mental health, and psychological inflexibility relative to the waitlist condition at posttreament; 3) improvements in psychological inflexibility would mediate the effects of ACT versus waitlist on health-related outcomes; and 4) participants would maintain gains at 8-week follow up in the ACT condition.

\section{Methods}

\section{Participants}

The sample consisted of 79 adults who are overweight/obese and interested in improving their diet and physical activity. Recruitment was conducted through university extension offices throughout the state. The study was framed as "testing out an online course to improve diet, exercise, and well-being." Participants reported learning about the study from sources including a friend $(n=15)$, extension newsletter $(n=14)$, extension faculty $(n=10)$, reddit community post $(n=10)$, employer email $(n=9)$, and extension social media/website $(n=9)$.

Participants were required to have a self-reported body mass index (BMI) of 25 or greater and regular access to internet. The majority of participants were white $(92.4 \%)$, female $(82.3 \%)$, and working full time $(83.5 \%)$. The average age was $39.56(\mathrm{SD}=12.12)$ and the average BMI was $33.78(\mathrm{SD}=5.69)$. Participants reported primarily participating to lose weight $(\mathrm{n}=45)$, to improve their diet $(n=38)$, to improve physical activity $(n=14)$, and/or to receive gift card incentives $(n=12)$. For more information on demographics see Table 1 .

\section{Procedures}

This clinical trial was pre-registered (NCT03932994) and was approved by the authors' Institutional Review Board. All study procedures were completed online (on Canvas or 
Qualtrics) or over the phone. Participants received gift cards for completing each assessment.

Participants first completed an initial screener and consent form on Qualtrics; if they were found eligible, they were then directed to an online baseline survey. After the survey participants were directed to complete one Automated Self-Administered 24-Hour Recall (ASA-24; see Measures section) [20]. Once all initial questionnaires had been completed, participants were randomized to participate in the ACT on Health online course or a waitlist.

Participants assigned to the ACT on Health course were instructed to complete one of eight sessions each week. Program usage was monitored by a doctoral student in clinical/counseling psychology who also provided weekly coaching calls to each participant, unless they indicated preference for emails. Coaching calls were brief, 5-10 minute phone calls, designed to increase adherence to the program and provide participants with support in implementing the program in their daily life (e.g., strategies to use, answer clarifying questions) The coaching protocol was based on an established protocol [21] that has been tested in prior guided self-help ACT studies for weight-related concerns [14]. After eight weeks, participants completed an online posttreatment survey, followed by three ASA-24 dietary recalls completed over the course of one week. Participants in the waitlist condition received access to ACT on Health after the posttreatment survey. A follow up survey was sent eight weeks after the posttreatment survey, followed by another series of three ASA-24 dietary recalls completed over a week.

\section{ACT on Health online intervention}

The 8-session ACT on Health course was delivered through Canvas, an online learning management system. Content was presented through a combination of text, videos, and interactive exercises. The program integrated ACT skills with nutrition education and strategies to increase physical activity. Sessions typically included education on the importance of a given 
food group, behavioral strategies to increase consumption of that given food group, strategies to increase physical activity, ACT skills to decrease cognitive affective barriers and increase intrinsic motivation for behavior change, and goal setting for the week that combined ACT and dietary/physical activity educational content (see Table 2). Over time, sessions increasingly integrated ACT skills and nutrition/physical activity content (e.g., linking diet/physical activity goals to values starting in session 3; focusing on mindful eating and movement in session 6).

\section{Measures}

Primary outcome. The Automated Self-Administered 24-Hour Recall (ASA24 [20]) was used as the primary outcome measure for healthy eating. The ASA-24 is an online selfadministered dietary recall measure in which participants report all food and drink consumed from "midnight to midnight" of the previous day. A Healthy Eating Index (HEI) score is calculated as a measure of dietary quality based on the Dietary Guidelines for Americans [22]. Scores are calculated in relation to ideal standards for several key food group per 1,000 calories consumed. HEI scores range from 0-100, with higher scores indicating greater consumption of healthy foods (e.g., fruits and vegetables) and lower consumption of unhealthy foods (e.g., added sugars and saturated fats). The average HEI in the US is 59 and diets that meet guidelines for a healthy diet would range from 74 to 100 [23]. Each 24-hour recall was reviewed for outliers (i.e., daily calories below $600 / 650$ or above $4400 / 5700$ for men and women respectively), which were removed prior to analyses. When multiple ASA-24 recalls were collected (at posttreatment and follow up), HEI scores were calculated per time point and averaged into a composite score. If only one ASA-24 was completed, that score was used for the HEI at that time point.

Secondary outcomes. Secondary measures of eating behaviors included the Three Factor Eating Questionnaire (TFEQ [24]), which assessed cognitive restraint (efforts to control food 
intake), uncontrolled eating (loss of control during eating), and emotional eating (eating in response to emotions), and the dietary choice subscale of the Weight Control Strategies Scale (WCSS [25]), which assessed eating behaviors that support weight loss. Physical activity measures included the physical activity subscale of the WCSS [25] and the International Physical Activity Questionnaire (IPAQ [26]), which assesses weekly physical activity by life domain and intensity as well as sedentary time. Total physical activity IPAQ scores were calculated in the standardized unit of Metabolic Equivalent Task (MET) in minutes per week. METs were scored for each domain by intensity of exercise, after truncating time in each to a maximum of 3 hours to reduce outliers, and were then summed into a total score. Participants self-reported their weight as a secondary outcome with the question "What is your current weight (please enter numerically in pounds)?" General mental health was assessed with the General Health Questionnaire (GHQ [27]). Weight self-stigma was assessed with the Weight Self-stigma Questionnaire (WSSQ [28]). Psychological inflexibility was assessed as the ACT process of change using the Acceptance and Action Questionnaire for Weight (AAQ-W [29]). The System Usability Scale (SUS [30]) assessed program usability and user acceptability.

Internal consistency for all the study measures that had a standard scoring method was adequate to excellent (TFEQ CR $\alpha=.74$, TFEQ UE $\alpha=.90$, TFEQ EE $\alpha=.88$; WCSS-DC $\alpha=$ .87 , WCSS-PA $\alpha=.92 ;$ GHQ $\alpha=.89$; AAQW $\alpha=.89$; SUS $\alpha=.83$ ).

\section{Data analysis plan}

This study initially aimed to recruit a sample of 60 participants over 5 months. However, the target sample was recruited within 12 days, and the target sample size was increased to 79 participants, who were enrolled over 28 days (between May 2019 and June 2019). This provided adequate power (.80) for an analysis of covariance (ANCOVA) to detect a between condition 
effect size of $d=.66$ at posttreatment controlling for baseline score using the sample of 74 participants who completed the posttreatment assessment.

Completer analyses were conducted with available data using a listwise deletion approach. Descriptive statistics were examined for program usage and satisfaction to examine the acceptability of the ACT on Health program. ANCOVAs tested for significant between condition effects (ACT vs. Waitlist) at posttreatment on each health-related outcome controlling for their pretreatment score. Paired sample $t$-tests examined within condition effects from posttreatment to follow up in the ACT condition (to test whether improvements maintain or even continue from posttreatment to 8-week follow up).

Finally, a series of mediational analyses were conducted with the cross product of coefficients test using 1,000 bootstrap samples. Each model included pretreatment mediator and outcome variables as covariates, with posttreatment weight-related psychological inflexibility (AAQW) as the mediator for the effect of condition on outcome. Mediational analyses were conducted with all outcomes that had a significant between condition effect at posttreatment.

\section{Results}

\section{Preliminary analyses}

The GHQ and IPAQ total physical activity variables were highly skewed and kurtotic. A logarithmic transformation of the GHQ and square root transformation of the IPAQ was used to approximate a normal distribution at each time point. There were no significant differences between conditions on any demographics, outcome or process measures $(p>.05)$. Overall, $94 \%$ completed the posttreatment self-report survey, and $87 \%$ completed the posttreatment ASA survey (see Figure 1), with no differences between condition on completion rates ( $p s>.10)$.

\section{Program usage and satisfaction ratings}


ACT on Health session completion was objectively gathered through the Canvas platform. On average participants in the ACT condition completed 5.55 sessions out of $8(S D=$ 3.32). The majority (59\%) completed all 8 sessions, with $69 \%$ completing at least half of sessions, and $79 \%$ completing at least session 1 . None of the baseline outcome, process, or demographic variables were related to number of sessions completed except baseline general mental health $(\mathrm{GHQ}), r(37)=-.34, p=.03)$, such that better mental health was related to completing more sessions. The only frequently endorsed $(n>3)$ reason for not completing all sessions at posttreatment was not having enough time $(n=7)$.

Participants reported adequate program usability on the SUS $(M=76.25, S D=15.66)$, which is within the "good" range based on previous benchmark research [31]. Participants gave positive satisfaction ratings on individual items rated on a 6-point scale with 4 ("slightly agree") or higher indicating a positive response. This included items assessing overall satisfaction $(M=$ 4.97, $S D=.87)$, perceived helpfulness $(M=5.03, S D=.90)$, ease of use $(M=5.18, S D=.97)$, perceived fit $(M=4.74, S D=1.24)$, desire to use the program in the future $(M=4.85, S D=$ 1.16), and perceived helpfulness for others $(M=5.26, S D=.86)$. Participants slightly agreed on average that coaching calls were helpful $(M=4.21, S D=1.65)$, but also slightly agreed the program would have been just as helpful without coaching calls $(M=4.09, S D=1.44)$. A review of open ended responses to what participants liked least in ACT on Health highlighted common themes including that the program was difficult to use $(n=7)$, particularly because it was not mobile friendly $(n=3)$, was too boring or slow $(n=5)$, particularly videos $(n=2)$, and they did not like the nutrition education component $(n=3)$, particularly because it was too basic $(n=2)$.

\section{ACT vs. waitlist effects on health-related outcomes at posttreatment}


A series of ANCOVA tested for differences between the ACT and waitlist conditions at posttreatment on each outcome, controlling for respective pretreatment scores (see Table 3). Significant condition effects were found for dietary outcomes including 24-hour dietary recall (HEI), uncontrolled eating (TFEQ-UE), emotional eating (TFEQ-EE), and dietary choice (WCSS-DC), but not for cognitive restraint with eating (TFEQ-CR). Significant condition effects were also found for self-reported weight, weight self-stigma (WSSQ), general mental health (GHQ), and weight-related psychological inflexibility (AAQW). In each case, participants in the ACT condition improved more on health-related outcomes at posttreatment relative to the waitlist condition. No significant condition effects were found for any physical activity measures, including WCSS-PA, IPAQ total physical activity, or IPAQ sedentary time.

\section{Maintenance of treatment effects at 8-week follow up in the ACT condition}

Paired sample $t$-tests examined whether improvements in health-related outcomes at posttreatment in the ACT condition were maintained or further improved at 8-week follow up (Table 3). The only two significant changes from posttreatment to follow up in the ACT condition were for dietary recall (HEI), which worsened to near baseline scores at follow up, and cognitive restraint with eating (TFEQ-CR), which improved from posttreatment to follow up.

\section{Psychological inflexibility mediation analyses}

A series of mediational analyses were conducted with the cross product of coefficients test to examine weight-related psychological inflexibility (AAQW) as a mediator of the effects of condition on outcomes at posttreatment (see Table 4). Changes in psychological inflexibility at posttreatment mediated the effects of ACT versus waitlist at posttreatment on uncontrolled eating (TFEQ-UE), emotional eating (TFEQ-EE), weight self-stigma (WSSQ), and general mental health (GHQ). In each case, the mediating pathway accounted for $51 \%$ to $91 \%$ of the 
variance in treatment on outcomes. However, psychological inflexibility did not mediate effects for dietary recall (HEI), dietary choice (WCSS-DC) or self-reported weight, which appeared in all cases to be due to a non-significant b-path (i.e., changes in psychological inflexibility were not related to changes in healthy eating or weight).

\section{Discussion}

This study evaluated the acceptability and efficacy of an online guided self-help program that integrates ACT with nutrition education for improving diet, physical activity, and other health-related outcomes among adults who are overweight/obese. Results indicated adequate acceptability based on program engagement (an average of 5.5 out of 8 sessions completed across participants) and self-reported program satisfaction. Participants assigned to ACT on Health improved more than the waitlist on healthy eating, self-reported weight, general mental health, weight self-stigma, and psychological inflexibility, but not self-reported physical activity. Changes in psychological inflexibility mediated improvements in some outcomes from ACT on Health, although not the primary dietary recall outcome or self-reported weight. Improvements in most outcomes were maintained at 8-week follow up in the ACT on Health condition, but not for the primary dietary recall outcome. Overall, these results provide some support for online guided self-help that integrates ACT with nutrition education for improving healthy eating, weight, and weight self-stigma, but highlight limitations in at least the current program for improving physical activity and maintaining some gains.

This study found that a guided self-help program combining ACT with nutrition education led to improvements in eating behaviors including overall healthy eating based on dietary recall, eating behaviors that support weight loss, emotional eating, and uncontrolled eating. For the primary outcome, average HEI scores in the ACT condition improved to 58, 
which approximated the average HEI of 59 in the US population [23], although this was still about one standard deviation below HEI scores consistent with meeting guidelines for a healthy diet [23]. In addition to their contribution to weight management, improving healthy eating can have direct effects on health outcomes and mortality [2]. Given the challenges of maintaining long term weight loss [6], targeting behaviors such as healthy eating independent of weight loss may benefit from increased emphasis in health promotion interventions [17]. A heavy emphasis on diet to only reduce weight might also risk reinforcing weight stigmatizing messages and link motivations for healthy eating to only weight loss, which might ultimately hinder sustainable dietary changes [17,32]. ACT may be particularly applicable as it focuses on clarifying intrinsic motivators for behavior change that are distinct from particular outcomes (e.g., weight loss), while decreasing maladaptive motivators for changing diet that lead to setting unrealistic goals that cannot be achieved or sustained, and ultimately lead to avoidance (e.g., weight self-stigma).

That said, this study found that although some eating behavior changes sustained at follow up, the primary outcome of healthy eating worsened. This is problematic given theorizing that ACT would support long-term behavior change, and this study only used a short term follow up assessment of 8 weeks. Prior studies have found long-term improvements when ACT is delivered in an in-person format $[8,10]$, but long-term effects have not been studied with selfguided ACT. Similarly, psychological inflexibility failed to mediate the effects of ACT on the primary healthy eating outcome due to a non-significant relation between psychological inflexibility and this measure. Prior ACT RCTs have found that changes in psychological inflexibility correlate with changes in binge eating [12]. However, it may be that psychological inflexibility applies more to maladaptive eating patterns like binge eating, rather than broader patterns of healthy eating as measured by the HEI. It may also be that ACT applies more directly 
to maladaptive eating patterns, which are regularly associated with thoughts and feelings, and that further programmed generalization of ACT skills is needed for the more day-to-day dietary behaviors and choices navigated for broader patterns of healthy eating.

This study also adds to a growing literature indicating ACT can reduce weight selfstigma among adults who are overweight/obese [11,14]. Furthermore, changes in psychological inflexibility were found to mediate the effects of ACT on weight self-stigma, which replicates prior findings from in-person interventions [11] in the context of an online intervention. Theoretically, learning to respond to stigma-related thoughts and feelings just for what they are, without acting on them, fighting with them, or otherwise responding to them as if they are literally true (i.e., decreased psychological inflexibility), is likely to reduce the process of selfstigmatization. This is important given research indicating that weight self-stigma has been found to reduce adherence to health behavior change interventions $[17,18]$.

Surprisingly, participants in the ACT on Health condition did not improve on any selfreported physical activity measures relative to the waitlist. Prior studies have found ACT to improve physical activity, measured objectively or through self-report, when delivered through book [14], app [15], and DVD formats [16]. One potential explanation was the relatively weak self-report assessment approach for measuring physical activity. For example, there was a high degree of within condition variability on IPAQ scores, which may have limited power to detect differences between conditions. Another potential explanation was that the ACT on Health program focused much more on nutrition education than physical activity, the latter of which was typically composed of just a few pages in each session aiming to increase motivation, identify new ways of exercising, or set goals for physical activity. Thus, the lack of effects on physical activity relative to healthy eating might be representative of the focus of intervention 
content. Finally, only a minority of participants (18\%) reported participating primarily to improve physical activity, with most interested in improving diet (48\%) and weight (57\%). It is not clear if ACT on Health would be sufficient in a study/sample focused on physical activity.

This study combined an online self-help intervention with weekly phone coaching. Coaching focused on increasing adherence to the online course, given challenges with engagement in purely self-guided programs and the superior outcomes when including coaching [19]. Yet, coaching also introduces limitations for scalability, and thus reach, for an otherwise highly scalable self-guided intervention, given the additional resources required per end user for a coach. Recent research has suggested more mixed findings and marginal benefits for coaching when directly comparing online programs with or without coaching [33]. The current study found relatively high engagement rates relative to purely self-guided ACT trials for health behavior change [15], which might be attributable in part to coaching. Similarly, participants reported being satisfied with coaching calls. However, participants also reported that coaching was not necessary. This matches our anecdotal experiences delivering coaching, in which at least a subset of participants did not appear to benefit from or need calls. It may be that as online programs continue to improve, coaching may be less necessary to promote engagement. In either case, future research might evaluate ACT on Health, or similar programs, without coaching given its added benefits for scalability and reach as a purely self-guided intervention.

There were notable limitations in this study that might reduce its generalizability and potential replicability. The most noteworthy limitation was with regards to measurement. The study primarily relied on self-report, which introduces substantial bias in assessing health outcomes such as physical activity and weight. Although the dietary recall method with the ASA-24 is a more rigorous assessment method, ideally three time points would have been 
gathered at baseline to match best practices and the number of assessments at other time points. However, we elected to only require a single dietary recall given the potential for participant attrition and lower engagement if participants had to wait a week before starting ACT on Health. Relatedly, the study did not include a long-term follow up assessment. Ideally this study would have included a between group comparison at follow up, with assessments of a year or more. The assessment schedule also limited mediational analyses due to being unable to test temporal relations between variables. Since the mediator and outcome were only assessed at pretreatment and posttreatment potential causal inferences from the mediation analyses are quite limited. The sample consisted of primarily White female participants and future research is needed to evaluate ACT on Health with males and minority groups. Finally, the ACT on Health program included a combination of factors that may account for positive effects relative to a waitlist condition (e.g., coaching calls, nutrition education, ACT, placebo, demand characteristics). Ideally ACT on Health would be evaluated in the future in such a way that the direct benefits of ACT could be determined while controlling for these other factors (e.g., testing dietary education alone versus dietary education plus ACT; testing ACT on Health with or without phone coaching).

This study provides further evidence that ACT can be effective for improving health outcomes among adults who are overweight/obese even when delivered in a more scalable, online guided self-help format. Integrating ACT with dietary education was effective at targeting key psychological variables for health behavior change such as psychological inflexibility and weight self-stigma. Further research is needed to determine the long-term effects of ACT when delivered in a self-help format, whether supplemental coaching is needed to ensure adequate engagement and outcomes, and how to further optimize online ACT interventions to maximize their reach and effectiveness. 


\section{References}

1. Kopelman, P. (2007). Health risks with overweight and obesity. Obesity Reviews, 8, S13-S17.

2. Wang, D. D., Li, Y., Chiuve, S. E., Hu, F. B., \& Willett, W. C. (2015). Improvements in US diet helped reduce disease burden and lower premature deaths, 1999-2012; overall diet remains poor. Health Affairs, 34, 1916-1922.

3. Warburton, D.E.R., Nicol, C.W. \& Bredin, S.S.D. (2006). Health benefits of physical activity: the evidence. Canadian Medical Association Journal, 174, 801-809.

4. Forman, E. M., \& Butryn, M. L. (2015). A new look at the science of weight control: how acceptance and commitment strategies can address the challenge of selfregulation. Appetite, 84, 171-180.

5. Gatewood, J. G., Litchfield, R. E., Ryan, S. J., Myers Geadelmann, J. D., Pendergast, J. F., \& Ullom, K. K. (2008). Perceived barriers to community-based health promotion program participation. American Journal of Health Behavior, 32, 260-271.

6. Loveman, E., Frampton, G. K., Shepherd, J., Picot, J., Cooper, K., Bryant, J., . . Clegg, A. (2011). The clinical effectiveness and cost-effectiveness of long-term weight management schemes for adults: a systematic review. Health Technology Assessment.

7. Hayes, S. C., Strosahl, K. D., \& Wilson, K. G. (2011). Acceptance and Commitment Therapy: The process and practice of mindful change. New York: The Guilford Press.

8. Lawlor, E.R., Islam, N., Bates, S., Griffin, S.J., Hill, A.J., Hughes, C.A., Sharp, S.J. \& Ahern, A.L. (2020). Third-wave cognitive behaviour therapies for weight management: A systematic review and network meta-analysis. Obesity Reviews, 21, e13013. 
9. Manchon, J., Quiles, M.J., Leon, E.M. \& Lopez-Roig, L. (2020). Acceptance and commitment therapy on physical activity: A systematic review. Journal of Contextual Behavioral Science, 17, 135-143.

10. Forman, E. M., Butryn, M. L., Manasse, S. M., Crosby, R. D., Goldstein, S. P., Wyckoff, E. P. \& Thomas, J. G. (2016). Acceptance-based versus standard behavioral treatment for obesity: Results from the mind your health randomized controlled trial. Obesity, 24, 2050-2056.

11. Lillis, J., Hayes, S. C., Bunting, K., \& Masuda, A. (2009). Teaching acceptance and mindfulness to improve the lives of the obese: A preliminary test of a theoretical model. Annals of Behavioral Medicine, 37, 58-69.

12. Afari, N., Herbert, M.S., Godfrey, K.M., Cuneo, J.G., Salamat, J.S., Mostoufi, S., ... Wetherell, J.L. (2019). Acceptance and commitment therapy as an adjunct to the MOVE! programme: a randomized controlled trial. Obesity Science \& Practice, 5, 397-407.

13. Tang, J.C., Abraham, C., Greaves, C.J. \& Nikolau, V. (2016). Self-directed interventions to promote weight loss: a systematic review and meta-analysis. Health Psychology Review, 10.

14. Potts, S., Krafft, J. \& Levin, M.E. (under review). A randomized controlled trial of acceptance and commitment therapy self-help with or without phone coaching for overweight and obese adults high in weight self-stigma.

15. Levin, M.E., Krafft, J., Seifert, S. \& Lillis, J. (In Press). Tracking valued and avoidant functions with health behaviors: A randomized controlled trial of the acceptance and commitment therapy matrix mobile app. Behavior Modification. 
16. Moffitt, R., \& Mohr, P. (2015). The efficacy of a self-managed acceptance and commitment therapy intervention DVD for physical activity initiation. British Journal of Health Psychology, 20, 115-129.

17. Mensinger, J.L., Calogero, R.M. \& Tylka, T.L. (2016). Internalized weight stigma moderates eating behavior outcomes in women with high BMI participating in a healthy living program. Appetite, 102, 32-43.

18. Mensinger, J.L. \& Meadows, A. (2017). Internalized weight stigma mediates and moderates physical activity outcomes during a healthy living program for women with high body mass index. Psychology of Sport and Exercise, 30, 64-72.

19. Andersson, G. (2016). Internet-delivered psychological treatments. Annual Review of Clinical Psychology, 12, 157-179.

20. Subar, A. F., Kirkpatrick, S. I., Mittl, B., Zimmerman, T. P., Thompson, F. E., Bingley, C., ... \& Potischman, N. (2012). The automated self-administered 24-hour dietary recall (ASA24): A resource for researchers, clinicians, and educators from the National Cancer Institute. Journal of the Academy of Nutrition and Dietetics, 112(8), 1134-1137.

21. Duffecy, J., Kinsinger, S., Ludman, E., \& Mohr, D. (2011). Telephone coaching to support adherence to internet interventions (TeleCoach): Coach Manual.

22. Krebs-Smith, S. M., Pannucci, T. E., Subar, A. F., Kirkpatrick, S. I., Lerman, J. L., Tooze, J. A., ... \& Reedy, J. (2018). Update of the healthy eating index: HEI-2015. Journal of the Academy of Nutrition and Dietetics, 118(9), 1591-1602.

23. Wilson, M.M., Reedy, J., \& Krebs-Smith, S.M. (2016). American diet quality: Where it is, where it is heading, and what it could be. Journal of the Academic of Nutrition and Dietetics, 116, 302-310. 
24. Stunkard, A. J., \& Messick, S. (1985). The three-factor eating questionnaire to measure dietary restraint, disinhibition and hunger. Journal of Psychosomatic Research, 29, 71-83.

25. Pinto, A. M., Fava, J. L., Raynor, H. A., LaRose, J. G., \& Wing, R. R. (2013). Development and validation of the weight control strategies scale. Obesity, 21, 2429-2436.

26. Booth, M. (2000). Assessment of physical activity: An international perspective. Research Quarterly for Exercise and Sport, 71(sup2), 114-120.

27. Werneke, U., Goldberg, D. P., Yalcin, I., \& Üstün, B. T. (2000). The stability of the factor structure of the General Health Questionnaire. Psychological Medicine, 30(4), 823-829.

28. Lillis, J., Luoma, J. B., Levin, M. E., \& Hayes, S. C. (2010). Measuring weight self-stigma: The weight self-stigma questionnaire. Obesity, 18(5), 971-976.

29. Lillis J. \& Hayes S.C. (2008). Measuring avoidance and inflexibility in weight-related problems. International Journal of Behavioral Consultation and Training, 4, 30-40.

30. Brooke, J. (1996). SUS: a "quick and dirty"e usability scale. In P.W. Jordan, B. Thomas, B.A. Weerdmeester, and I.L. McClelland (Eds.) Usability Evaluation in Industry (189-194).

31. Bangor, A., Kortum, P. T., \& Miller, J. T. (2008). An empirical evaluation of the System Usability Scale. International Journal of Human-Computer Interaction, 24, 574-594.

32. Tylka, T.L., Annunziato, R.A., Burgard, D., Daníelsdottir, S., Shuman, E., Davis, C., \& Calogero, R.M. (2014). The weight-inclusive versus weight-normative approach to health: evaluating the evidence for prioritizing well-being over weight loss. Journal of Obesity, 1e18.

33. Shim, M., Mahaffey, B., Bleidistel, M. \& Gonzalez, A. (2017). A scoping review of humansupport factors in the context of Internet-based psychological interventions (IPIs) for depression and anxiety disorders. Clinical Psychology Review, 57, 129-140. 
Table 1. Participant demographics by condition.

\begin{tabular}{|c|c|c|}
\hline & $\begin{array}{l}\text { ACT on Health } \\
\qquad(N=39)\end{array}$ & $\begin{array}{l}\text { Waitlist } \\
(N=40)\end{array}$ \\
\hline Body Mass Index at time 1 (SD) & $34.01(6.5)$ & $33.48(4.9)$ \\
\hline Age (SD) & $38.05(9.4)$ & $41.03(14.3)$ \\
\hline \multicolumn{3}{|l|}{ Gender $(\%)$} \\
\hline Female & 82.1 & 82.5 \\
\hline Male & 17.9 & 17.5 \\
\hline \multicolumn{3}{|l|}{ Ethnicity (\%) } \\
\hline Hispanic/Latinx & 2.6 & 10.0 \\
\hline Not Hispanic/Latinx & 97.4 & 90.0 \\
\hline \multicolumn{3}{|l|}{ Race $(\%)^{*}$} \\
\hline White & 89.7 & 95.0 \\
\hline Asian & 2.6 & 0 \\
\hline Black & 2.6 & 7.5 \\
\hline Native Hawaiian or other Pacific Islander & 5.1 & 0 \\
\hline Median household income & $\$ 60,000-79,000$ & $\$ 60,000-79,000$ \\
\hline \multicolumn{3}{|l|}{ Employment status (\%) } \\
\hline Full time & 84.6 & 82.5 \\
\hline Part time & 7.7 & 15.0 \\
\hline Student & 0 & 2.5 \\
\hline Unemployed & 2.6 & 0 \\
\hline Stay at home parent & 5.1 & 0 \\
\hline \multicolumn{3}{|l|}{ Marital status (\%) } \\
\hline Married & 53.8 & 52.5 \\
\hline Living with someone as if married & 10.3 & 12.5 \\
\hline Widowed & 5.1 & 5.0 \\
\hline Divorced or annulled & 7.7 & 5.0 \\
\hline Separated & 0 & 2.5 \\
\hline Never married & 23.1 & 22.5 \\
\hline \multicolumn{3}{|l|}{ Education (\%) } \\
\hline High school or equivalent & 5.1 & 5.0 \\
\hline Some college & 15.4 & 20.0 \\
\hline College or technical degree & 15.4 & 20.0 \\
\hline Bachelor's degree & 38.5 & 30.0 \\
\hline Master's or specialist degree & 20.5 & 25.0 \\
\hline Doctoral degree & 5.1 & 0 \\
\hline
\end{tabular}

*Participants were allowed to choose multiple categories. 
Table 2. Outline of ACT on Health content.

Session

1. Starting the program

2. Watching your thinking

3. Finding your motivation

4. Acknowledging

\& opening up

5. Moving forward

6. Being mindful

7. Building patterns

8. Continuing your journey
Diet/Physical Activity Content

Balanced eating; Reviewing MyPlate

plan; Setting small eating goal.

Increasing fruits and vegetables;

Recommended physical activity guidelines; Goal setting for physical activity and eating fruits/vegetables.

Increasing wholegrains; Identifying motivators for exercise; Valuesbased goal setting for physical activity and whole grains.

Reducing high calorie/low nutritional value foods; Practicing acceptance with physical activity.

Increasing lean protein sources; Reducing sedentary behaviors; Values-based goal setting for sedentary behavior and eating.

Mindless eating; Mindfulness of hunger and fullness cues; Practicing mindful eating; Goal setting for mindful movement and eating.

Meal planning; Grocery shopping; Saving time in the kitchen; Eating choice points; Values-based goal setting for physical activity.

Reviewing health behavior changes and maintaining gains.
ACT Content

Introducing the ACT approach - How people get stuck with weight stigma and unrealistic weight/behavior change goals; Compassionate behavior change.

Defusing from unhelpful thoughts - Mind as an unhelpful, harsh coach; Noticing thoughts as thoughts; Leaves on a stream mindfulness exercise.

Identifying values - Distinguishing values from unhelpful motivators (e.g., stigma, "shoulds"); Values card sort; Linking values to behavior change.

Introducing acceptance - Costs of avoidance; Breaking avoidance patterns; Urge surfing.

Practicing acceptance and cognitive defusion - Breaking unhelpful rules; Welcoming unwanted guests exercise; Labeling meditation.

Being mindful of the present - Costs of running on autopilot; Practicing mindfulness; Mindfulness with eating and physical activity.

Committed action - Building larger patterns of valued action; Values-based goal setting in non-health domains; Noticing choice points for values versus avoidance.

Committed action - Values as a compass; Recommitting after slips; Reviewing ACT skills, Writing a letter to the future you. 
Table 3. Descriptive statistics, ANCOVA, and t-test results.

\begin{tabular}{|c|c|c|c|c|c|c|c|c|}
\hline & \multicolumn{2}{|c|}{ Waitlist Condition } & \multicolumn{3}{|c|}{ ACT Condition } & \multicolumn{2}{|c|}{$\underline{\mathrm{ACT}}$ vs. Waitlist Post } & \multirow{2}{*}{$\frac{\text { ACT Post-Follow }}{\text { Paired } t \text {-test }}$} \\
\hline & $\begin{array}{l}\text { Pre } \\
M(S D)\end{array}$ & $\begin{array}{l}\text { Post } \\
M(S D)\end{array}$ & $\begin{array}{l}\text { Pre } \\
M(S D)\end{array}$ & $\begin{array}{l}\text { Post } \\
M(S D)\end{array}$ & $\begin{array}{l}\text { Follow Up } \\
M(S D)\end{array}$ & $\overline{\mathrm{ANCOVA} F}$ & $\overline{\text { partial }} \eta^{2}$ & \\
\hline \multicolumn{9}{|l|}{ Eating Behavior Outcomes } \\
\hline Healthy Eating (ASA-HEI) & $\begin{array}{l}48.93 \\
(11.21)\end{array}$ & $\begin{array}{l}50.74 \\
(10.60)\end{array}$ & $\begin{array}{l}51.60 \\
(13.97)\end{array}$ & $\begin{array}{l}57.66 \\
(12.12)\end{array}$ & $\begin{array}{l}52.27 \\
(9.64)\end{array}$ & $5.38 *$ & .08 & $-2.30^{*}$ \\
\hline Uncontrolled Eating (TFEQ-UE) & $\begin{array}{l}21.23 \\
(6.12)\end{array}$ & $\begin{array}{l}21.18 \\
(5.92)\end{array}$ & $\begin{array}{l}22.36 \\
(5.42)\end{array}$ & $\begin{array}{l}20.23 \\
(4.80)\end{array}$ & $\begin{array}{l}19.80 \\
(5.48)\end{array}$ & $6.04 *$ & .08 & .25 \\
\hline Emotional Eating (TFEQ-EE) & $\begin{array}{l}8.23 \\
(2.82)\end{array}$ & $\begin{array}{l}8.18 \\
(2.71)\end{array}$ & $\begin{array}{l}8.23 \\
(2.48)\end{array}$ & $\begin{array}{l}7.31 \\
(2.40)\end{array}$ & $\begin{array}{l}6.86 \\
(2.06)\end{array}$ & $4.07 *$ & .05 & 1.28 \\
\hline Cognitive Restraint (TFEQ-CR) & $\begin{array}{l}13.10 \\
(3.14)\end{array}$ & $\begin{array}{l}13.90 \\
(3.10)\end{array}$ & $\begin{array}{l}14.38 \\
(3.16)\end{array}$ & $\begin{array}{l}14.60 \\
(2.61)\end{array}$ & $\begin{array}{l}15.71 \\
(2.77)\end{array}$ & .00 & .00 & $2.60 *$ \\
\hline Dietary Choice (WCSS-DC) & $\begin{array}{l}29.35 \\
(8.18)\end{array}$ & $\begin{array}{l}30.59 \\
(7.48)\end{array}$ & $\begin{array}{l}30.41 \\
(8.10)\end{array}$ & $\begin{array}{l}34.80 \\
(5.57)\end{array}$ & $\begin{array}{l}35.37 \\
(6.59)\end{array}$ & $11.99 * *$ & .14 & .23 \\
\hline \multicolumn{9}{|l|}{ Physical Activity Outcomes } \\
\hline Physical Activity (WCSS-PA) & $\begin{array}{l}13.28 \\
(6.96)\end{array}$ & $\begin{array}{l}14.56 \\
(7.49)\end{array}$ & $\begin{array}{l}14.59 \\
(6.44)\end{array}$ & $\begin{array}{l}17.34 \\
(5.03)\end{array}$ & $\begin{array}{l}18.23 \\
(5.22)\end{array}$ & 3.35 & .05 & .56 \\
\hline Physical Activity (IPAQ-PA) & $\begin{array}{l}4185.70 \\
(5228.37)\end{array}$ & $\begin{array}{l}4546.54 \\
(4543.38)\end{array}$ & $\begin{array}{l}4299.73 \\
(4532.63)\end{array}$ & $\begin{array}{l}5453.29 \\
(4598.09)\end{array}$ & $\begin{array}{l}4580.13 \\
(4029.52)\end{array}$ & 1.64 & .02 & -.94 \\
\hline Sedentary Time (IPAQ) & $\begin{array}{l}3104.10 \\
(1262.07)\end{array}$ & $\begin{array}{l}2586.74 \\
(1265.37)\end{array}$ & $\begin{array}{l}3200.77 \\
(1290.30)\end{array}$ & $\begin{array}{l}2788.20 \\
(1227.20)\end{array}$ & $\begin{array}{l}2620.00 \\
(1268.68)\end{array}$ & -.21 & .00 & 1.21 \\
\hline \multicolumn{9}{|l|}{ Other Secondary Outcomes } \\
\hline Weight in lbs. & $\begin{array}{l}204.03 \\
(33.30)\end{array}$ & $\begin{array}{l}201.94 \\
(35.88)\end{array}$ & $\begin{array}{l}214.10 \\
(44.60)\end{array}$ & $\begin{array}{l}208.23 \\
(39.19)\end{array}$ & $\begin{array}{l}207.48 \\
(43.57)\end{array}$ & $4.60 *$ & .06 & .83 \\
\hline Weight Self-Stigma (WSSQ) & $\begin{array}{l}37.83 \\
(10.30)\end{array}$ & $\begin{array}{l}37.33 \\
(10.01)\end{array}$ & $\begin{array}{l}34.85 \\
(10.12)\end{array}$ & $\begin{array}{l}30.60 \\
(10.04)\end{array}$ & $\begin{array}{l}30.66 \\
(10.22)\end{array}$ & $8.77 * *$ & .11 & -.22 \\
\hline Mental Health (GHQ) & $\begin{array}{l}26.63 \\
(6.30)\end{array}$ & $\begin{array}{l}26.03 \\
(5.39)\end{array}$ & $\begin{array}{l}25.05 \\
(5.57)\end{array}$ & $\begin{array}{l}22.63 \\
(5.87)\end{array}$ & $\begin{array}{l}22.77 \\
(5.69)\end{array}$ & $6.37^{*}$ & .08 & -.72 \\
\hline Psych. Inflexibility (AAQW) & $\begin{array}{l}82.45 \\
(20.51)\end{array}$ & $\begin{array}{l}83.31 \\
(22.50)\end{array}$ & $\begin{array}{l}73.28 \\
(20.88)\end{array}$ & $\begin{array}{l}63.89 \\
(18.42)\end{array}$ & $\begin{array}{l}63.46 \\
(19.23)\end{array}$ & $13.82 * * *$ & .16 & .82 \\
\hline
\end{tabular}

${ }^{*} p<.05 ; * * p<.01 ; * * * p<.001$. TFEQ-UE, TFEQ-EE, IPAQ Sitting, weight, WSSQ, GHQ, and AAQW are scored such that lower scores indicate better health. TFEQ-CR, WCSS-DC, WCSS-PA, and IPAQ-PA are scored such that higher scores indicate better health. Descriptive statistics are calculated with all available data and with non-transformed scores. ANCOVAs test condition effects (ACT vs. Waitlist) at posttreatment controlling for respective pretreatment score. 
Table 4. Mediation analysis results testing weight-related psychological inflexibility as a mediator of treatment outcomes.

\begin{tabular}{|c|c|c|c|c|c|c|c|}
\hline & \multirow{2}{*}{$\frac{\text { a path }}{X-M}$} & \multirow{2}{*}{$\frac{\text { b path }}{M(X)-Y}$} & \multirow{2}{*}{$\frac{\text { c path }}{X-Y}$} & \multirow{2}{*}{$\frac{c^{\prime} \text { path }}{X(M) Y}$} & \multicolumn{3}{|c|}{ Products of coefficients } \\
\hline & & & & & $\begin{array}{l}\text { Point } \\
\text { estimate (SE) }\end{array}$ & $\begin{array}{l}\text { Bootstrapping } \\
95 \% \text { CI }\end{array}$ & $\begin{array}{l}\text { Proportion } \\
\text { mediated }\left(1-c^{\prime} / c\right)\end{array}$ \\
\hline ASA-HEI & $4.22 * * *$ & .72 & $2.13 *$ & 1.55 & $1.03(1.43)$ & $-1.75,3.89$ & \\
\hline TFEQ-UE & $3.70 * * *$ & $4.36^{* * *}$ & $2.01 *$ & .30 & $1.41(.46)$ & $.66,2.51$ & $85 \%$ \\
\hline TFEQ-EE & $3.71 * * *$ & $2.99 * *$ & 1.15 & -.10 & $.50(.23)$ & $.13,1.09$ & $91 \%$ \\
\hline WCSS-DC & $3.72 * * *$ & 1.49 & $3.35 * *$ & $2.49 *$ & $.71(.50)$ & $-.02,2.02$ & \\
\hline Weight & $3.69 * * *$ & 1.19 & $3.68 *$ & 1.70 & $.82(.60)$ & $-.15,2.34$ & \\
\hline WSSQ & $4.52 * * *$ & $4.76^{* * *}$ & $3.00 * *$ & .76 & $3.65(1.21)$ & $1.52,6.40$ & $75 \%$ \\
\hline GHQ & $3.72 * * *$ & $3.10 * *$ & $2.67 * *$ & 1.32 & $.02(.01)$ & $.01, .05$ & $51 \%$ \\
\hline
\end{tabular}

${ }^{*} p<.05 ;{ }^{* *} p<.01 ; * * *<.001 . \mathrm{X}-\mathrm{M}=$ effect of treatment condition (ACT vs. Waitlist) on mediator (AAQW), $\mathrm{M}(\mathrm{X})-\mathrm{Y}=$ relation between mediator and outcome controlling for treatment condition, $\mathrm{X}-\mathrm{Y}=$ effect of treatment condition on outcome, $\mathrm{X}(\mathrm{M}) \mathrm{Y}=$ remaining effect of treatment condition on outcome after controlling for mediator. $t$-test values are reported for paths tested. 
Figure 1. Participant flow

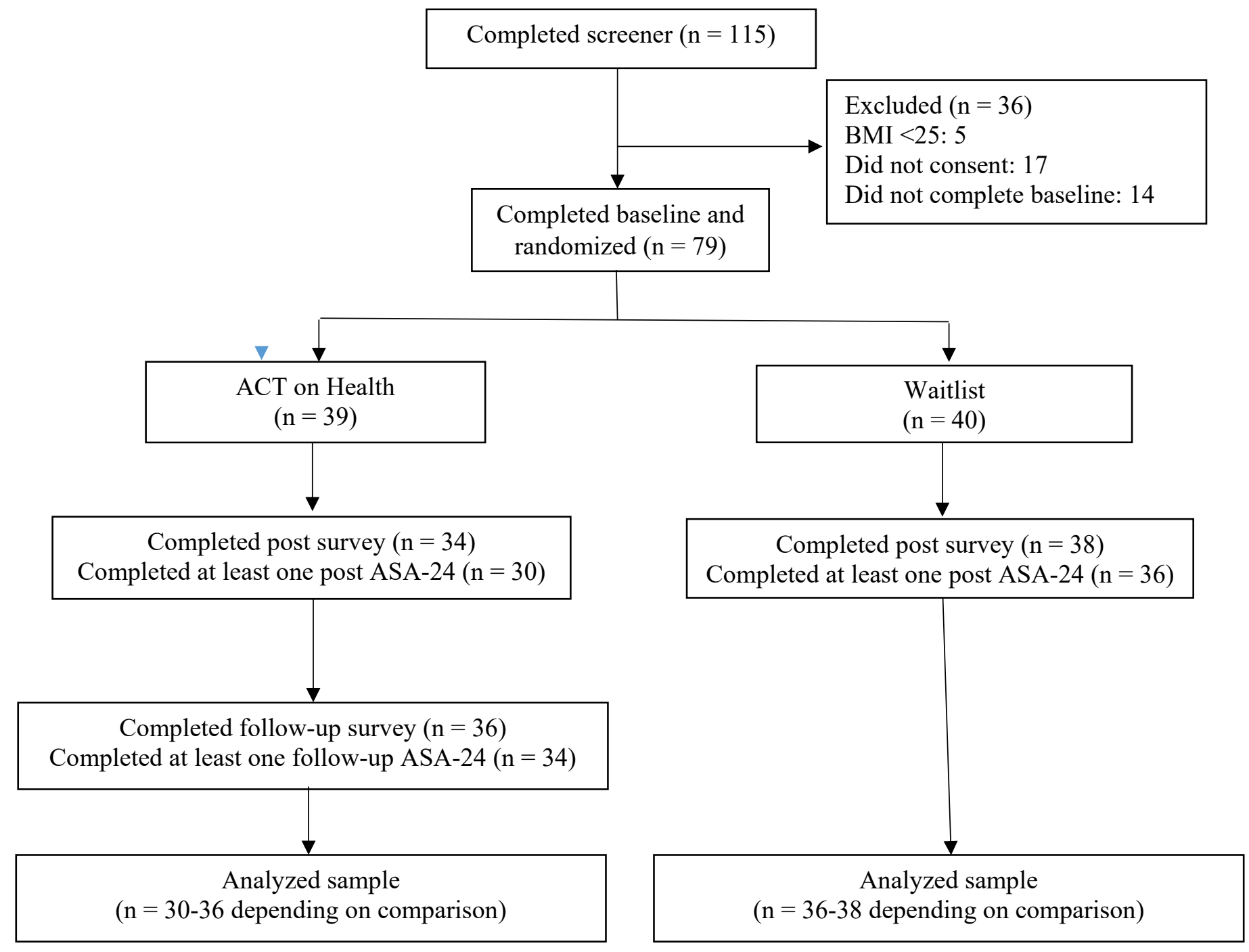

